\title{
Unified Constitutive Equations of Viscoplastic Deformation: Development and Capabilities*
}

\author{
Kwnagsoo $\mathrm{HO}^{* *}$
}

\begin{abstract}
This paper addresses a viscoplastic constitutive model that allows a consistent way of modeling positive and negative rate sensitivities of flow stress, which is associated with dynamic strain aging occurring in a certain regime of loading rates and temperatures. Based on the concept of continuum mechanics, a phenomenological constitutive model includes the use of a yield surface within the framework of unified viscoplastic constitutive equations. An extension of modeling capability to negative rate sensitivity of flow stress is accomplished through a rate-dependent format of nonlinear kinematic hardening rule that causes the back stress to be rate-dependent. The negative rate sensitivity of the back stress enable to predict the influence of prior strain rate on relaxation behavior, which means that the relaxed stress of the fastest prior strain rate has the smallest magnitude at the end of relaxation period.
\end{abstract}

Key Words: Constitutive Equations, Viscoplasticity, Dynamic Strain Aging, Relaxation, Negative Rate Sensitivity

\section{Introduction}

The inelastic deformation behavior of metals and alloys is in general considered to be rate-dependent even at low homologous temperature. As the temperature increases the rate dependency of material deformation behavior is recognized as being predominant. In the past several decades a number of constitutive laws have been developed to model a wide range of inelastic deformation behavior under various loading conditions. Major classes of these modeling efforts have introduced the concept of internal state variables, which represent the changing characteristics of microstructure with inelastic deformation. Moreover, they have not separated rate-independent plasticity and rate-dependent creep within the framework of unified viscoplastic constitutive laws and thus have used only one inelastic strain to describe inelastic deformation processes with or without the concept of yield surface $^{(1)-(5)}$.

In a certain range of strain, strain rate and temperature, metallic materials and alloys exhibit serrated flow referred to as Portevin-Le Chatelier (PLC) effect, which is related to inhomogeneous deformation. This phenomenon is associated with negative strain rate sensitivity that is a

\footnotetext{
* Received 22nd March, 2005 (No. 05-5027)
}

** Department of Mechanical Engineering, Keimyung University, 1000 Sindang-Dong, Dalseo-Gu, Daegu 704-701, Korea(South). E-mail: hok@kmu.ac.kr result of dynamic strain aging due to the interaction of diffusing solute atoms with mobile dislocations. Considerable research efforts have been devoted to microscopic mechanisms responsible for the PLC effect in conjunction with the experimental exploration of the domain where the PLC effect is present ${ }^{(6)-(9)}$.

In the context of the unified viscoplastic constitutive laws, a great deal of theoretical work has been performed to model the rate-dependent responses, such as strain hardening, creep, ratcheting and stress relaxation, to loading over a wide range of loading rates, temperatures and other loading conditions. In fact, the model development has been focused on the nonlinear, inelastic and ratedependent deformation behavior associated with positive strain rate sensitivity of flow stress, namely an increase of flow stress with loading rate. The achievements have provided the improvement of design and safe operation by stress analyses of structural components and manufacturing processes. It should be noted, however, that there still exists a necessity for extending the modeling capabilities to the inelastic behavior concerned with negative rate sensitivity when material experiences dynamic strain aging for a certain range of temperature.

The present paper aims at contributing to a phenomenological method of modeling negative rate sensitivity (flow stress decreases with an increase of loading rate) and rate-insensitivity (flow stress is independent of loading rate) as well as positive rate sensitivity within the 
framework of unified viscoplastic constitutive laws using a yield surface. To this end, the flow stress in fully developed inelastic deformation region is decomposed into the plastic contribution of rate independency and the viscous one of rate dependency. A mathematical classification of the three types of rate sensitivity, i.e., positive, zero (rateinsensitivity) and negative rate sensitivities, is thus feasible from a phenomenological point of view.

\section{Framework of the Constitutive Equations}

\subsection{Flow law}

Numerous experimental and theoretical studies have been conducted to investigate microscopic mechanisms associated with dynamic strain aging. Unfortunately, the work appears to be incomplete concerning strain rate effect on stress-strain curve and various rate-dependent inelastic deformations as global material responses, and thus may not be appropriate for the use of inelastic stress analysis of structural components and manufacturing processes. On the other hand, the unified viscoplasic models by Miller and Sherby ${ }^{(10)}$ and Yaguchi and Takahashi ${ }^{(11)}$ predicted negative strain rate sensitivity of the flow stress on the stress-strain curve.

The present formulation introduces the yield condition of a von Mises type based on the concept of Perzyna $^{(12)}$ and Chaboche ${ }^{(13)}$ models that may be understood as a generalization of the classical theory of the plasticity. Previously, the viscoplasticity theory based on overstress without a yield surface had been developed to reproduce inelastic deformation behavior including negative strain rate sensitivity, see Ho and $\mathrm{Krempl}^{(14)}$ and $\mathrm{Ho}^{(15)}$. Using a yield criterion, which is contrasted with the previous versions, the proposed model as a further refinement has an excellent correlation with the elastic limit and satisfies the generalized normality law concerned with a dissipation potential in fully developed flow stress region.

On the assumption of small strain, volume preserving inelastic deformation and isotropy the three dimensional constitutive equations on isothermal condition consist of the volumetric and deviatoric flow law, and the evolution laws of the state variables. Total deviatoric strain rate is assumed to be the sum of elastic component $\dot{e}_{i j}^{e l}$ and inelastic component $\dot{e}_{i j}^{i n}$ :

$$
\dot{e}_{i j}=\dot{e}_{i j}^{e l}+\dot{e}_{i j}^{i n}
$$

where $e_{i j}=\varepsilon_{i j}-\frac{1}{3} \delta_{i j} \varepsilon_{k k}$ is the deviatoric component of the strain $\varepsilon_{i j}$. The elastic component obeys the rate form of Hooke's law:

$$
\dot{e}_{i j}^{e l}=\frac{1+v}{E} \dot{s}_{i j}
$$

where $E$ and $v$ are the elastic modulus and Poisson's ratio, respectively. $s_{i j}$ is the deviatoric component of the true stress $\sigma_{i j}$ and a superposed dot denotes material time derivative. Next, the inelastic component is newly defined as

$$
\dot{e}_{i j}^{i n}=\frac{3}{2} B\left\langle\frac{J\left(\sigma_{i j}-H_{i j}\right)-R}{D}\right\rangle^{m} \frac{\left(s_{i j}-g_{i j}\right)}{J\left(\sigma_{i j}-G_{i j}\right)}
$$

where $B$ and $m$ are material constants. $H_{i j}$ and $R$ denote the kinematic hardening and the isotropic hardening variable, respectively, and $D$ represents the drag stress that is considered to be constant in the present formulation. $\langle>$ is Macauley's bracket and means that: $\langle x\rangle=x$ if $x>0$ and $<x>=0$ if $x \leq 0$. The yield criterion of a von Mises type is expressed by the kinematic and isotropic hardenings as

$$
f=J\left(\sigma_{i j}-H_{i j}\right)-R \leq 0
$$

Using the second invariant, $J\left(\sigma_{i j}-H_{i j}\right)$ and $J\left(\sigma_{i j}-G_{i j}\right)$ are defined as follows:

$$
\begin{aligned}
& J\left(\sigma_{i j}-H_{i j}\right)=\sqrt{\frac{3}{2}\left(s_{i j}-h_{i j}\right)\left(s_{i j}-h_{i j}\right)} \\
& J\left(\sigma_{i j}-G_{i j}\right)=\sqrt{\frac{3}{2}\left(s_{i j}-g_{i j}\right)\left(s_{i j}-g_{i j}\right)}
\end{aligned}
$$

where $h_{i j}$ and $g_{i j}$ are the deviators of the state variables $H_{i j}$ and $G_{i j}$, respectively. The volumetric deformation is elastic as follows:

$$
\dot{\varepsilon}_{k k}=\frac{1-2 v}{E} \dot{\sigma}_{k k}
$$

The use of a yield criterion enables to define the pure elastic domain. It is worthy to mention that the constitutive equations admit long-time asymptotic solutions thought to correspond to fully established inelastic flow. The asymptotic limit of $J\left(\sigma_{i j}-H_{i j}\right)-R$ in Eq. (3) reduces to the overstress invariant $J\left(\sigma_{i j}-G_{i j}\right)$. Thus the inelastic strain rate in asymptotic state becomes a function of the overstress, which is the difference between the stress $\sigma_{i j}$ and the back stress $G_{i j}$; details are discussed in section 2.3.

\subsection{Evolution equations for state variables}

The evolution equation for the difference between the state variables $g_{i j}$ and $h_{i j}$ is expressed by a nonlinear kinematic hardening format attributed to Armstrong and Frederick $^{(16)}$. The evolution law thus represents the competing effect of strain hardening and dynamic recovery:

or

$$
\left(\dot{g}_{i j}-\dot{h}_{i j}\right)=\psi\left[\frac{2}{3} \dot{e}_{i j}^{i n}-\frac{\left(g_{i j}-h_{i j}\right)}{R} \dot{\phi}\right]
$$

$$
\dot{g}_{i j}=\psi\left[\frac{2}{3} \dot{e}_{i j}^{i n}-\frac{\left(g_{i j}-h_{i j}\right)}{R} \dot{\phi}\right]+\dot{h}_{i j}
$$

where $\psi$ is a material constant that influences the transition between the pure elastic deformation and the fully established inelastic deformation. The effective inelastic strain rate is defined as follows:

$$
\dot{\phi}=\sqrt{\frac{2}{3} \dot{e}_{i j}^{i n} \dot{e}_{i j}^{i n}}=B\left\langle\frac{J\left(\sigma_{i j}-H_{i j}\right)-R}{D}\right\rangle^{m}
$$

The kinematic stress $H_{i j}$ is introduced with the objective of describing the strain hardening behavior in monotonic loading condition. The evolution of the kinematic stress is expressed by 


$$
\dot{h}_{i j}=\frac{2}{3} E_{t} \dot{e}_{i j}^{i n}
$$

where $E_{t}$ is the inelastic modulus at the maximum inelastic strain of interest. It is related to the inelastic modulus based on total strain $\hat{E}_{t}$ by $\hat{E}_{t}=E_{t} /\left(1+E_{t} / E\right)$.

The scalar variable $R$ with the dimension of stress is defined to be rate-dependent by utilizing the overstress invariant $J\left(\sigma_{i j}-G_{i j}\right)$ as follows:

$$
R=r_{c}+\left\langle A+\beta J\left(\sigma_{i j}-G_{i j}\right)\right\rangle
$$

where $r_{c}$ is a positive constant and $\beta<1$ is a model parameter. When $\beta \geq 1$, the constitutive equations model only elastic deformation behavior since inelastic deformation never occurs by virtue of the yield criterion. It will be shown later that the present constitutive laws are able to model positive, zero and negative rate sensitivity in a mathematically consistent way by introducing the parameter $\beta$. The purpose of the scalar state variable $A$ is to describe cyclic hardening or softening. It represents the change in the size of the elastic domain and its evolution is given by

$$
\dot{A}=A_{r}\left(A_{f}-A\right) \dot{\phi} ; \quad A(t=0)=A_{0}
$$

where $A_{r}$ and $A_{f}$ are material constants. $A_{r}$ indicates the evolving rate of the state variable $A$, and $A_{f}$ is an asymptotic value corresponding to the stabilized cyclic deformation behavior.

The evolution law of Eq. (7.a) provides a balance between the strain hardening and the dynamic recovery when fully established inelastic flow, namely saturation, is reached. In this situation, the difference $g_{i j}-h_{i j}$ depends on the rate-dependent variable $R$. Therefore the back stress deviator $g_{i j}$ becomes a rate-dependent form that is an essential feature of the present constitutive model enabling to model all types of strain rate sensitivity.

\subsection{Features of the constitutive laws}

The constitutive laws consist of a set of coupled differential equations. By converting these differential equations into integral representation and formally taking the limits for $t \rightarrow \infty$, one can obtain long-time asymptotic solutions for constant strain rate condition ${ }^{(15),(17)}$. Since the asymptotic solutions reveal basic features of the constitutive model, they provide useful guides for developing constitutive laws that have more accurate and versatile modeling capabilities for inelastic deformation behavior. In practice, the asymptotic limits are thought to correspond to material behavior in the regime of fully established inelastic flow with sufficient accuracy.

When the asymptotic limit is reached, the following relations hold all the time:

$$
\begin{aligned}
& \left\{\dot{s}_{i j}\right\}=\left\{\dot{g}_{i j}\right\}=\left\{\dot{h}_{i j}\right\} \\
& \{\dot{A}\}=0
\end{aligned}
$$

where \{\} means the asymptotic value of a quantity. Equation (12) shows that the state variables, $s_{i j}, g_{i j}$ and $h_{i j}$, evolve at the same rate in fully established inelastic flow. Therefore, the slope of the stress-strain curve in inelastic flow is attributed to the evolution of the state variable $h_{i j}$ that plays a role in setting the inelastic modulus. The scalar state variable $A$ is saturated and its asymptotic value is thus reached in inelastic flow region.

There exist the following asymptotic solutions for the differences between the state variables:

$$
\begin{aligned}
& \left\{g_{i j}-h_{i j}\right\}=\{R\} \frac{\left\{s_{i j}-g_{i j}\right\}}{\left\{J\left(\sigma_{i j}-G_{i j}\right)\right\}} \\
& \left\{s_{i j}-h_{i j}\right\}=\left\{R+J\left(\sigma_{i j}-G_{i j}\right)\right\} \frac{\left\{s_{i j}-g_{i j}\right\}}{\left\{J\left(\sigma_{i j}-G_{i j}\right)\right\}}
\end{aligned}
$$

Applying the asymptotic relation $\left\{\dot{g}_{i j}\right\}=\left\{\dot{h}_{i j}\right\}$ to Eq. (7.a), one arrives at Eq. (14). Equation (15) is then obtained by adding $\left\{s_{i j}-g_{i j}\right\}$ on both sides of Eq. (14).

Substituting the asymptotic solution for $\left\{s_{i j}-h_{i j}\right\}$ presented in Eq. (15) into Eq. (5.a) and rearranging, we obtain

$$
\left\{J\left(\sigma_{i j}-H_{i j}\right)\right\}=\left\{R+J\left(\sigma_{i j}-G_{i j}\right)\right\}
$$

Using Eqs. (3) and (16) we obtain an asymptotic solution for the inelastic strain rate:

$$
\left\{\dot{e}_{i j}^{i n}\right\}=\frac{3}{2} B\left\langle\frac{\left\{J\left(\sigma_{i j}-G_{i j}\right)\right\}}{D}\right\rangle^{m} \frac{\left\{s_{i j}-g_{i j}\right\}}{\left\{J\left(\sigma_{i j}-G_{i j}\right)\right\}}
$$

It is seen from Eqs. (14), (15) and (17) that the inelastic strain rate becomes a function of the overstress and thus the asymptotic differences, $\left\{g_{i j}-h_{i j}\right\}$ and $\left\{s_{i j}-h_{i j}\right\}$, are in the direction of the inelastic strain rate in the asymptotic state. Since the inelastic strain rate in the asymptotic state depends only on the overstress we may define a viscoplastic potential from which the normality law is satisfied.

\section{Capabilities of the Model}

To comprehend the framework of the constitutive equations, it is most effective to represent a specialization of the model for uniaxial loading. The multiaxial formulation presented in the previous section is thus reduced to the uniaxial form below. For uniaxial condition, $\sigma_{11}=\sigma$ is the only nonzero stress component and the tensorial state variables are all zero except for $G_{11}=G$ and $H_{11}=H$, so that we have from Eqs. (5.a) and (5.b): $J\left(\sigma_{i j}-G_{i j}\right)=|\sigma-G|$ and $J\left(\sigma_{i j}-H_{i j}\right)=|\sigma-H|$. Using $\varepsilon_{11}=\varepsilon$ and $\dot{A}=0$ for the sake of simplicity, the constitutive equations become

$$
\begin{aligned}
& \dot{\varepsilon}=\dot{\varepsilon}^{e l}+\dot{\varepsilon}^{i n}=\frac{\dot{\sigma}}{E}+B\left\langle\frac{|\sigma-H|-R}{D}\right\rangle^{m} \frac{(\sigma-G)}{|\sigma-G|} \\
& \dot{G}=\psi\left[\dot{\varepsilon}^{i n}-\frac{(G-H)}{R}\left|\dot{\varepsilon}^{i n}\right|\right]+\dot{H} \\
& \dot{H}=E_{t} \dot{\varepsilon}^{i n}
\end{aligned}
$$

The asymptotic solutions for the differences between the state variables are considered again under uniaxial loading so as to investigate the influence of the model parameter $\beta$ on inelastic deformation behavior. Equations (14) and (15) are reduced to 


$$
\begin{aligned}
& \{G\}=\{H\}+\left\{r_{c}+A+\beta|\sigma-G|\right\} \frac{\{\sigma-G\}}{\{|\sigma-G|\}} \\
& \{\sigma\}=\{H\}+\left\{r_{c}+A+(1+\beta)|\sigma-G|\right\} \frac{\{\sigma-G\}}{\{|\sigma-G|\}}
\end{aligned}
$$

We have made use of $\left\{g_{11}-h_{11}\right\}=(2 / 3)\left\{G_{11}-H_{11}\right\},\left\{s_{11}-\right.$ $\left.g_{11}\right\}=(2 / 3)\left\{\sigma_{11}-G_{11}\right\},\left\{s_{11}-h_{11}\right\}=(2 / 3)\left\{\sigma_{11}-H_{11}\right\}$ and Eq. (10) with $A+\beta J\left(\sigma_{i j}-G_{i j}\right)>0$ to arrive at these relations. It is seen from Eqs. (18.a) and (18.c) with $\{\dot{\sigma}\}=\{\dot{H}\}$ that the overstress invariant $\{|\sigma-G|\}$ is nonlinearly related to the strain rate, namely $\sqrt[m]{|\dot{\varepsilon}|}$, and the overstress in the asymptotic state thus increases with an increase in strain rate.

Equation (19) reveals that the back stress is ratedependent due to the overstress invariant multiplied by the model parameter $\beta$. When $\beta=0$, the back stress becomes rate-independent. In this case the model does not predict different relaxed stresses at the end of relaxation period with different prior strain rates. It will be shown later that the rate dependency of the back stress plays important role to describe proper relaxation behavior and dynamic strain aging.

In the asymptotic state, the flow stress consists of the rate-independent contribution $\left\{H+r_{c}+A\right\}$ and the ratedependent one $\{(1+\beta)|\sigma-G|\}$ as seen in Eq. (20). For $-1<\beta<1$ the rate-dependent contribution $\{(1+\beta)|\sigma-G|\}$ is nonlinearly increasing with strain rate and the flow stress thus increases with an increase in strain rate, which means positive rate sensitivity. When $\beta=-1$ is set the ratedependent contribution to the flow stress disappears and zero rate sensitivity is depicted. Finally, the condition $\beta<-1$ corresponds to negative rate sensitivity because the rate-dependent contribution decreases with an increase in strain rate. Therefore, using the mathematical classification by the model parameter $\beta$ the constitutive equations are capable of modeling the three types of rate sensitivity.

\subsection{Monotonic loading}

The rate-dependent deformation behavior is demonstrated by numerical experiments for a hypothetical material whose constants are arbitrarily chosen and given in Table 1 so as to show qualitative modeling capabilities. Unless indicated otherwise, the material constants of Table 1 are used in the modeling of inelastic deformation behavior for a hypothetical material.

Figures 1 (a) - (c) illustrate the influence of the model parameter $\beta$ on the stress, the back stress and the kinematic stress at constant strain rates ranging from $10^{-6} \mathrm{~s}^{-1}$ to $10^{-3} \mathrm{~s}^{-1}$. Based on the mathematical classification by the parameter $\beta$ discussed in the previous section, Fig. 1 (a) shows positive strain rate sensitivity by setting $\beta=-0.5$. And Fig. 1 (b) and (c) depict zero rate sensitivity for $\beta=-1$ and negative rate sensitivity for $\beta=-1.5$, respectively. It is seen from Eq. (19) that the back stress exhibits negative rate sensitivity due to the negative value of the parameter $\beta$ in all three cases. In contrast with the rate dependency
Table 1 Material constants

\begin{tabular}{llll}
\hline & Hypothetical mat. & $9 \mathrm{Cr}-1 \mathrm{Mo}$ & IN738LC \\
\hline$B\left[s^{-1}\right]$ & $3 \times 10^{-5}$ & $1 \times 10^{-1}$ & 1 \\
$D[\mathrm{MPa}]$ & 100 & 350 & 500 \\
$m$ & 15 & 12 & 17 \\
$E[\mathrm{MPa}]$ & $200 \times 10^{3}$ & $190 \times 10^{3}$ & $185 \times 10^{3}$ \\
$E_{t}[\mathrm{MPa}]$ & 1000 & 2300 & 4500 \\
$\beta$ & -0.5 & -1.0 & -1.2 \\
$r_{c}[\mathrm{MPa}]$ & 1 & 1 & 1 \\
$A_{r}$ & 0 & 0 & 0 \\
$A_{0}[\mathrm{MPa}]$ & 250 & 525 & 795 \\
$\psi[\mathrm{MPa}]$ & $100 \times 10^{3}$ & $400 \times 10^{3}$ & $100 \times 10^{4}$ \\
\hline & & &
\end{tabular}

of the back stress, the kinematic stress is shown to be rateindependent. The stress, the back stress and the kinematic stress grow at the same rate in the fully established inelastic flow region that starts from about $1 \%$ strain.

Figure 2 shows that the initial elastic limit changes with the magnitude of the scalar state variable $A$, which is taken here to be constant during deformation under the assumption of cyclic neutral behavior. Since the inelastic strain rate must be zero during the pure elastic deformation the back stress and the kinematic stress do not evolve with deformation. Thus, the elastic limit linearly depends on the magnitude of the $A$ as expected from Eqs. (10) and (18.a). As seen in Fig. 2, when the magnitude of $A$ increases the flow stress increases with an increase of the elastic limit, but the difference between the flow stresses for $10^{-5} \mathrm{~s}^{-1}$ and $10^{-3} \mathrm{~s}^{-1}$ strain rates is not changed. Figure 3 depicts the change of the slope of the stress-strain curve according to the change of the inelastic modulus. It is apparent from the figure that the stress and the back stress evolve at the very same rate as the kinematic stress in the fully established inelastic flow.

\subsection{Relaxation behavior}

It has been known that relaxation rate strongly depends on prior strain rate. For a constant relaxation time the relaxed stress associated with the fastest prior strain rate has the smallest magnitude at the end of the relaxation period $^{(11),(18),(19)}$. Figures 4 and 5 depict tensile tests followed by relaxation for different strain rates. It is seen from Eq. (19) that the back stress in Fig. 4 (a) for $\beta=-0.5$ is rate-dependent, whereas it is rate-independent when $\beta=0$ is used in Fig. 5 (a).

Using $\dot{\varepsilon}=0$, the stress rate during relaxation test can be obtained from Eq. (18.a) as follows:

$$
\dot{\sigma}=-E B\left\langle\frac{|\sigma-H|-R}{D}\right\rangle^{m} \frac{(\sigma-G)}{|\sigma-G|}
$$

It should be noted that the relaxation terminates when $|\sigma-H|=R$ is reached. At the beginning of the relaxation, the kinematic stresses for different prior strain rates have the same magnitude due to rate independency in loading. Then the differences among their change during relaxation may be neglected. Therefore, the relaxed stress at the ter- 


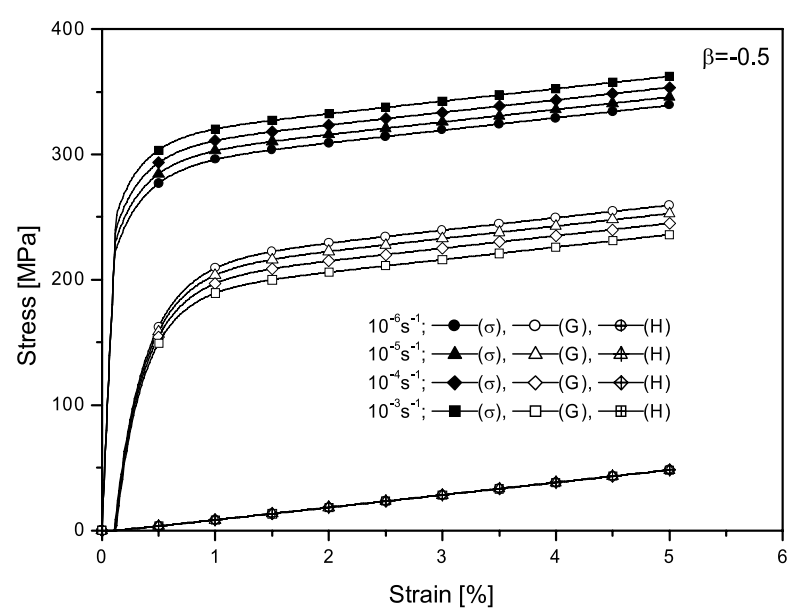

(a)

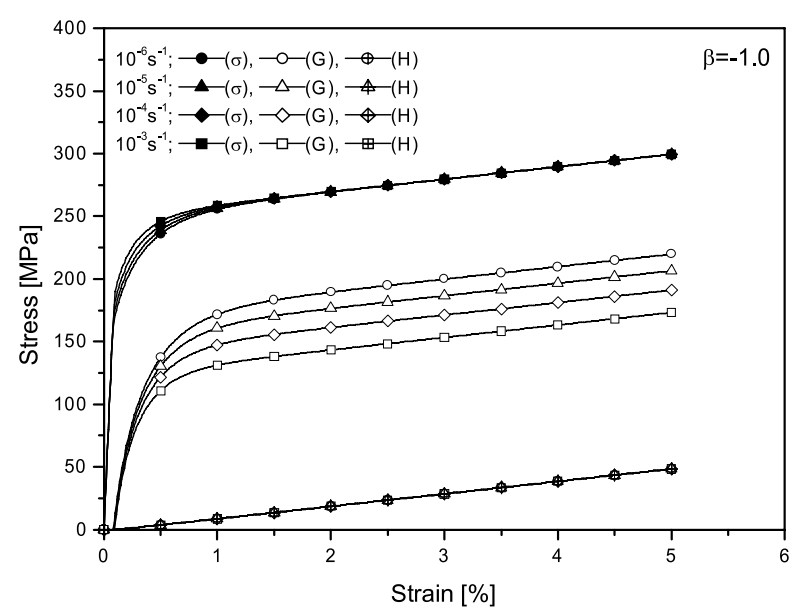

(b)

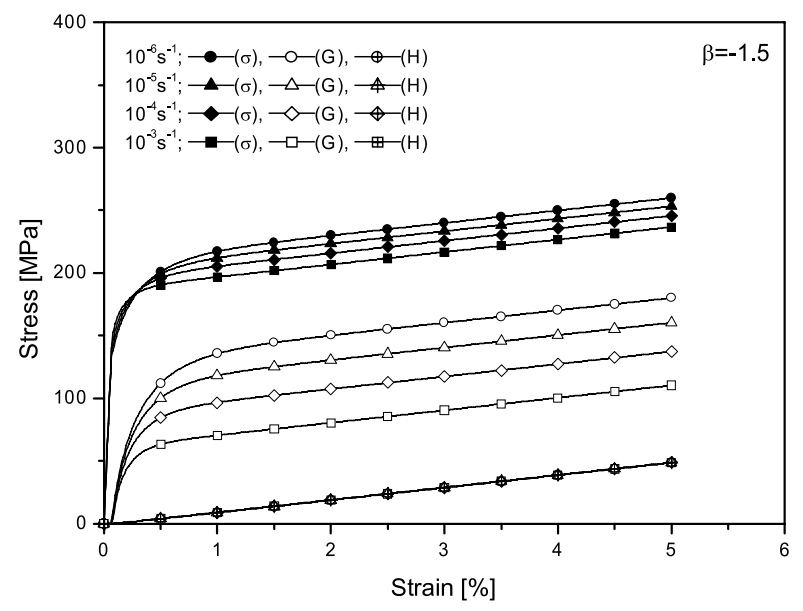

(c)

Fig. 1 The stress together with the back stress and the kinematic stress at constant strain rates are plotted: (a) positive rate sensitivity with $\beta=-0.5$, (b) zero rate sensitivity with $\beta=-1.0$, (c) negative rate sensitivity with $\beta=-1.5$

mination of the relaxation depends only on the variable $R$. From Eq. (10), the rate dependency of the variable $R$ dis-

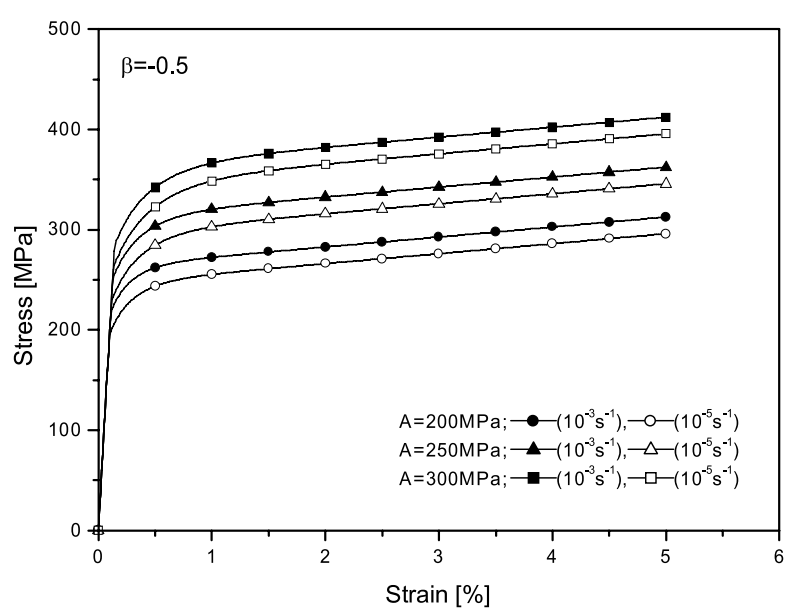

Fig. 2 The state variable $A$ is changed to examine its influence on the flow stress at the strain rates of $10^{-3} \mathrm{~s}^{-1}$ and $10^{-5} \mathrm{~s}^{-1}$

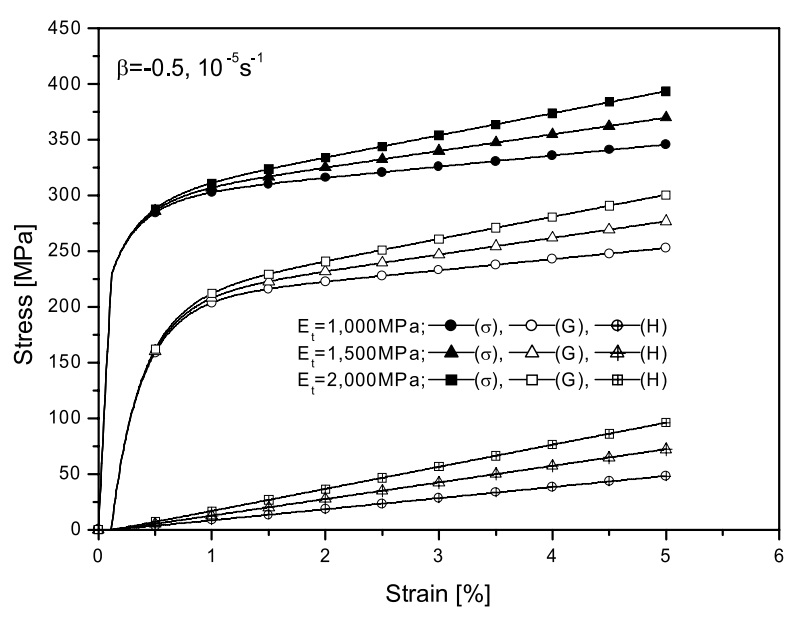

Fig. 3 Effect of the inelastic modulus $E_{t}$ at the strain rate of $10^{-5} \mathrm{~s}^{-1}$

appears for $\beta=0$. Accordingly, the relaxed stresses for the prior strain rates ranging from $10^{-6} \mathrm{~s}^{-1}$ to $10^{-4} \mathrm{~s}^{-1}$ finally end up at one point as seen in Fig. 5 (b), contradicted with experimental evidences in literature. On the other hand, when $\beta=-0.5$, the variable $R$ represents negative rate sensitivity that is possible only with a negative value of the parameter $\beta$, so that the model depicts the different endpoints of the relaxed stresses as shown in Fig. 4 (b).

\subsection{Strain rate jump effect}

The strain rate sensitivity of flow stress is in general investigated through strain rate change test in which two different strain rates are cycled. A strain rate jump results in the immediate and transient response followed by the quasi-steady one that corresponds to the monotonic stressstrain curve of the new strain rate ${ }^{(20)-(23)}$.

Schematic for a strain rate jump of $\dot{\varepsilon}_{1}<\dot{\varepsilon}_{2}$ is shown in Fig. 6 where two types of strain rate sensitivity may be defined. The instantaneous strain rate sensitivity $\left(S_{i}=\right.$ $\left.\Delta \sigma_{i} / \Delta \ln \dot{\varepsilon}\right)$ by an immediate stress jump $\Delta \sigma_{i}$ is positive 

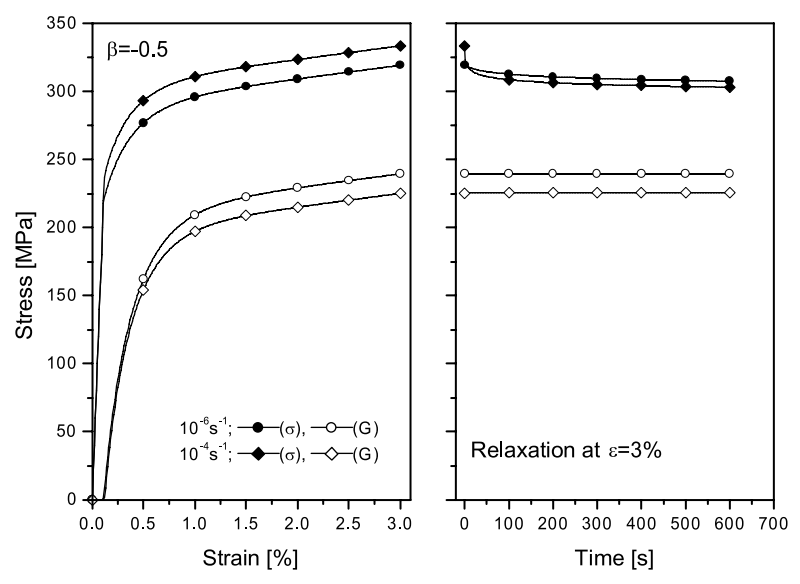

(a)

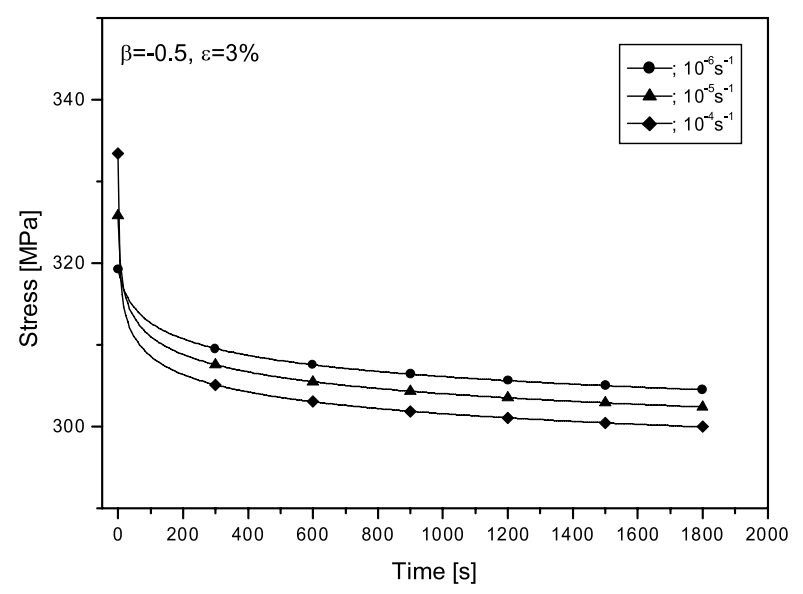

(b)

Fig. 4 Relaxation behavior with $\beta=-0.5$ : (a) stress-strain and subsequent relaxation curves, (b) stress-time curves for different prior strain rate. Each relaxation test starts at $3 \%$ strain

and then the negative, quasi-steady strain rate sensitivity $\left(S_{s}=\Delta \sigma_{s} / \Delta \ln \dot{\varepsilon}\right)$ develops due to dynamic strain aging after the transient behavior has been disappeared. Estrin and Kubin $^{(21)}$ explained the reasons for the transient behavior by the solute concentration on the dislocation that cannot immediately adjust to the new strain rate.

Figures 7 and 8 depict the responses to the strain rate cycling between $10^{-3} \mathrm{~s}^{-1}$ and $10^{-5} \mathrm{~s}^{-1}$ for $\beta=-1.0$ and -1.5 , respectively. In both cases, the instantaneous strain rate sensitivities are always shown to be positive. For $\beta=-1.0$, the quasi-steady strain rate sensitivity after positive or negative strain rate jumps becomes zero because of the rate independence of the flow stress, as seen in Fig. 7. In Fig. 8 for $\beta=-1.5$, each quasi-steady behavior rejoins the monotonic stress-strain curve corresponding to the new rate and its rate sensitivity exhibits negative. The present constitutive equations are competent to model the strain rate jump behavior that is characterized by both of the instantaneous and the quasi-steady rate sensitivities regardless of the strain rate sensitivity type of the flow stress.
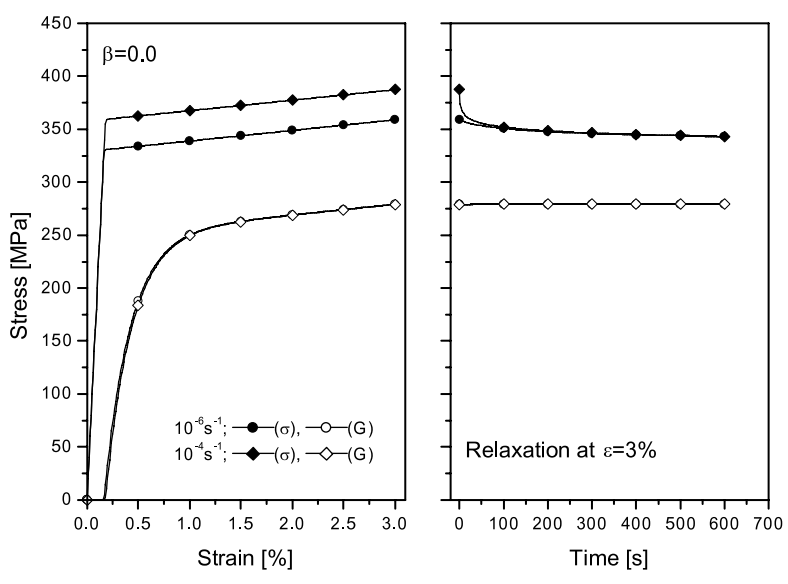

(a)

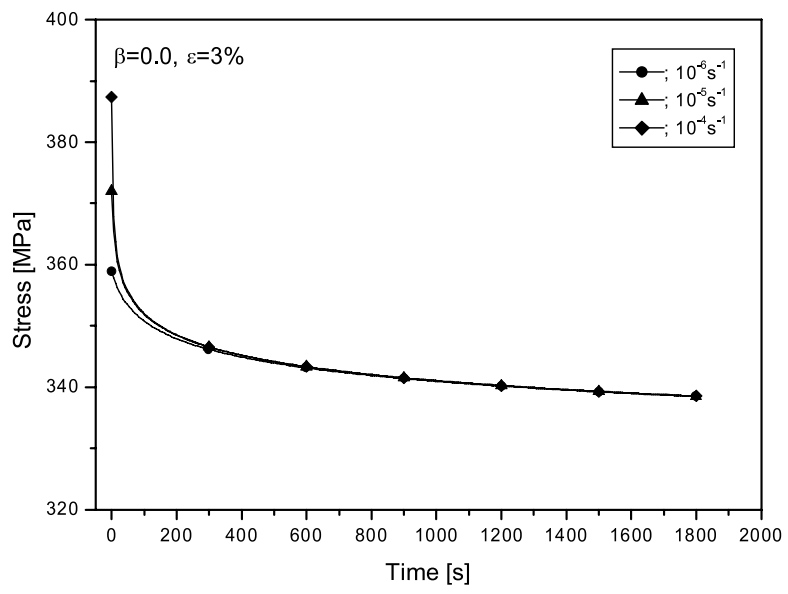

(b)

Fig. 5 Relaxation behavior with $\beta=0.0$ : (a) stress-strain and subsequent relaxation curves, (b) stress-time curves for different prior strain rate. Each relaxation test starts at $3 \%$ strain

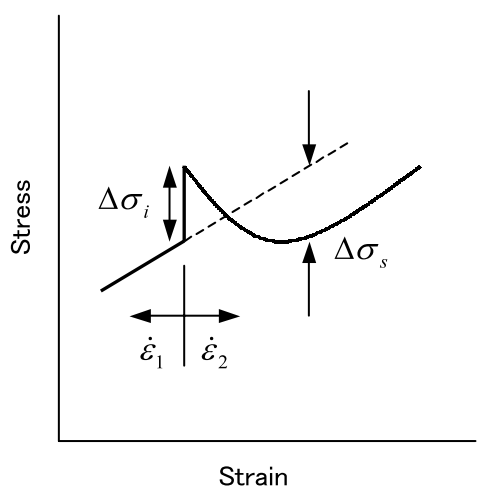

Fig. 6 Schematic showing a strain rate jump behavior under negative rate sensitivity of flow stress; $\dot{\varepsilon}_{1}<\dot{\varepsilon}_{2}$

\section{4 Applications}

The proposed constitutive equations are applied to simulate the experimental results of modified $9 \mathrm{Cr}-1 \mathrm{Mo}$ steel by Yaguchi and Takahashi ${ }^{(11)}$ and nickel-based superalloy IN738LC by Yaguchi et al. ${ }^{(24)}$ so as to evaluate the modeling capabilities of the constitutive equations. Us- 


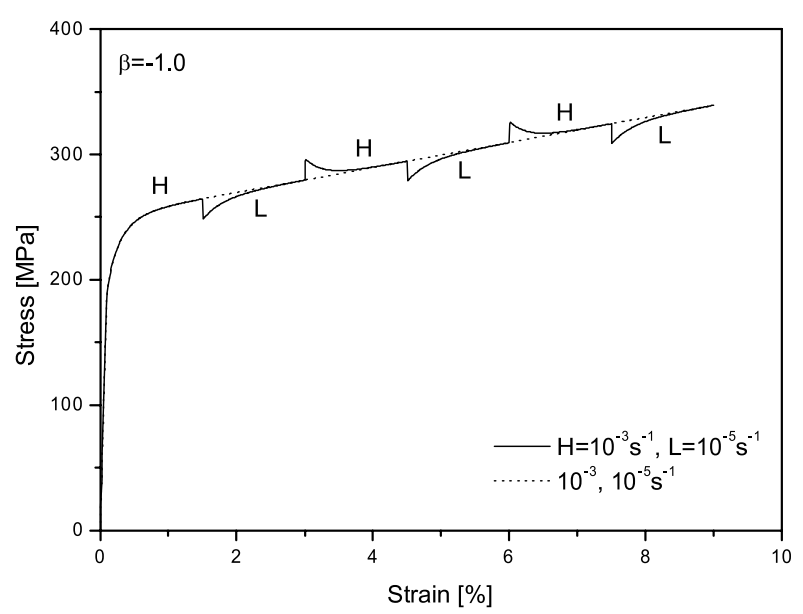

Fig. 7 Strain rate cycling behavior with the strain rate changing between $10^{-3} \mathrm{~s}^{-1}$ and $10^{-5} \mathrm{~s}^{-1}$. $\beta=-1.0$ is used for the zero rate sensitivity and the dotted curves at the constant strain rates of $10^{-3}$ and $10^{-5} \mathrm{~s}^{-1}$ are plotted

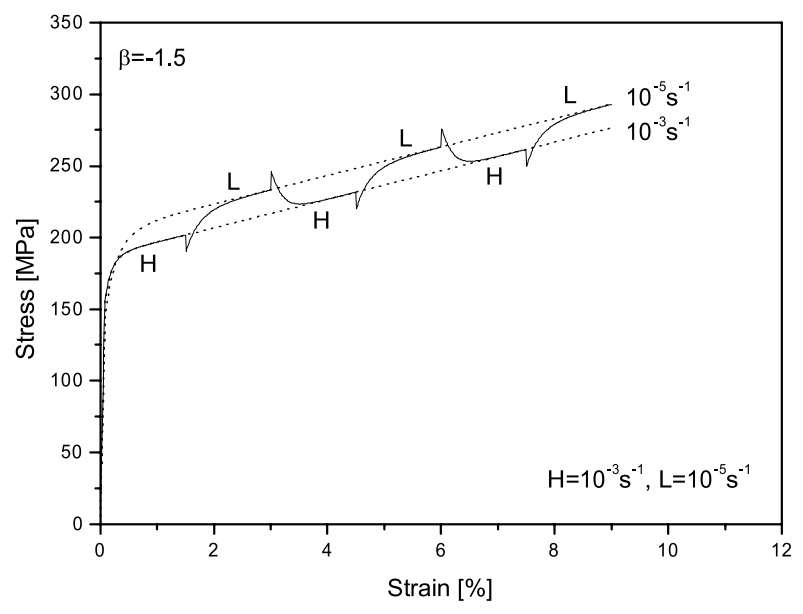

Fig. 8 Strain rate cycling behavior under the negative rate sensitivity using $\beta=-1.5$. As references, the dotted curves at the constant strain rates are also plotted

ing the material constants given in Table 1, Figs. 9 and 10 show the simulated results for modified $9 \mathrm{Cr}-1 \mathrm{Mo}$ steel at $400^{\circ} \mathrm{C}$ and IN738LC at $450^{\circ} \mathrm{C}$, respectively. The material constants are preliminarily determined by estimating the asymptotic solutions at a specific strain in the flow stress region for the experimental data from monotonic tensile and relaxation tests. Then, they are compensated by trial and error technique to produce good agreement between the calculated and the experimental data, see $\mathrm{Ho}^{(15)}$ for details.

As shown in Fig. 9, the flow stresses for the monotonic tensile loadings exhibit rate independence, which may be recognized as the influence of dynamic strain aging. The stress-strain curves at the strain rates of $10^{-6} \mathrm{~s}^{-1}$ and $10^{-3} \mathrm{~s}^{-1}$ show positive rate sensitivity in the transition region between the pure elastic deformation and the fully established inelastic flow, but they exhibit rate indepen-

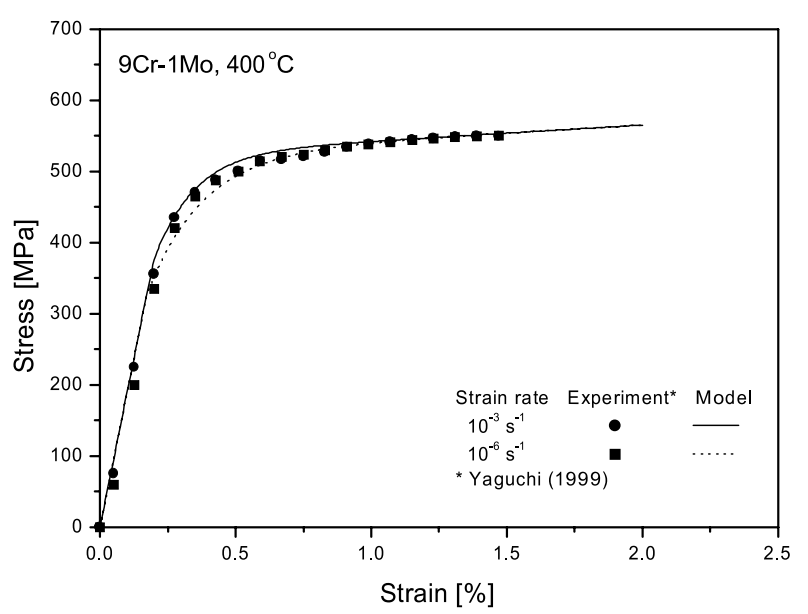

(a)

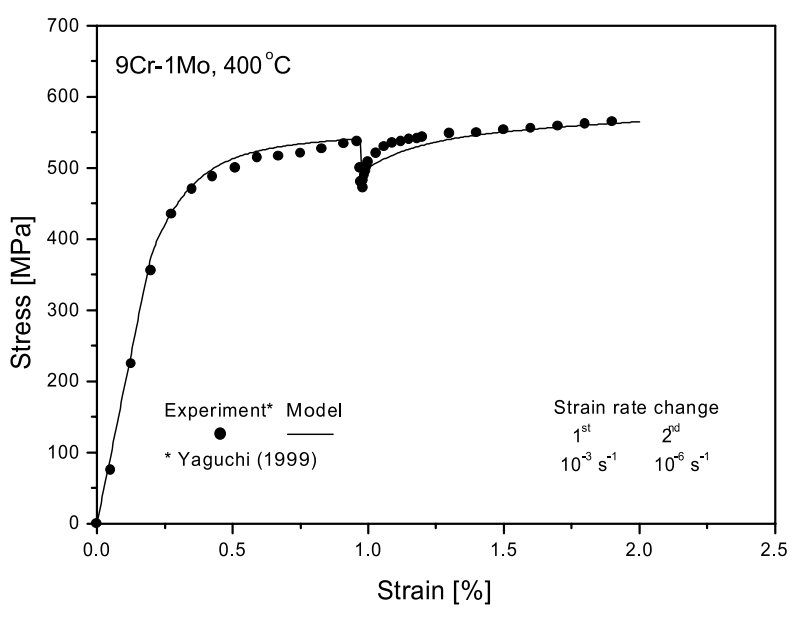

(b)

Fig. 9 Simulations of zero rate sensitivity for modified 9Cr$1 \mathrm{Mo}$ steel at $400^{\circ} \mathrm{C}$ : (a) stress-strain curve, (b) strain rate change behavior

dency over about $1 \%$ strain as seen in Fig. 9 (a). Referring to Fig. 9 (b), a strain rate jump from $10^{-3} \mathrm{~s}^{-1}$ to $10^{-6} \mathrm{~s}^{-1}$ results in an instantaneous stress drop meaning that the instantaneous strain rate sensitivity is positive. Then it gradually rejoins the stress-strain curve corresponding to the strain rate of $10^{-6} \mathrm{~s}^{-1}$ where the quasi-steady strain rate sensitivity becomes zero.

It can be seen in Fig. 10 (a) that the strain rate sensitivity in the flow stress region is slightly negative. The simulation describes small positive rate sensitivity in the transition, but it simulates well the experimental data in the flow stress region. The relaxation behavior at $\varepsilon=1.2 \%$ for two different prior strain rates, $10^{-6}$ and $10^{-3} \mathrm{~s}^{-1}$ is shown in Fig. 10 (b). At the termination of the relaxation test, the relaxed stress associated with $10^{-3} \mathrm{~s}^{-1}$ has a smaller magnitude.

\section{Discussion and Conclusions}

Within the framework of unified state variable theo- 


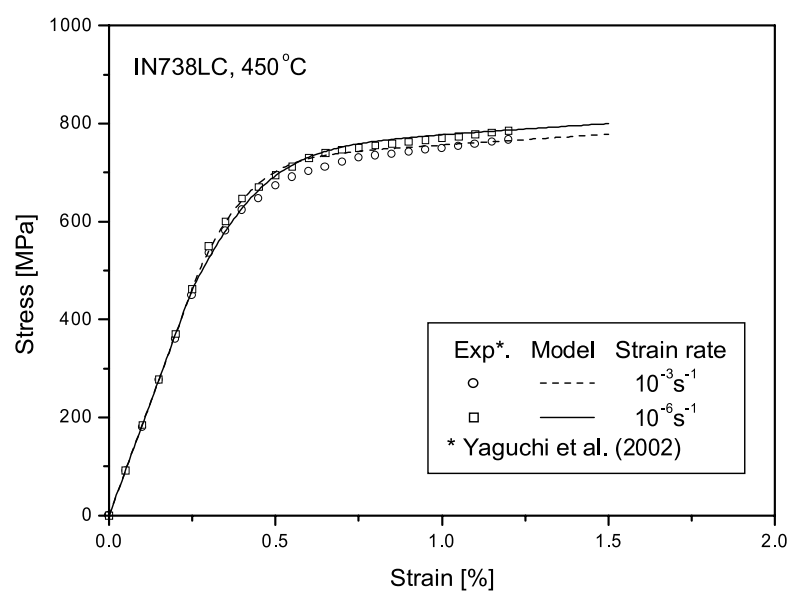

(a)

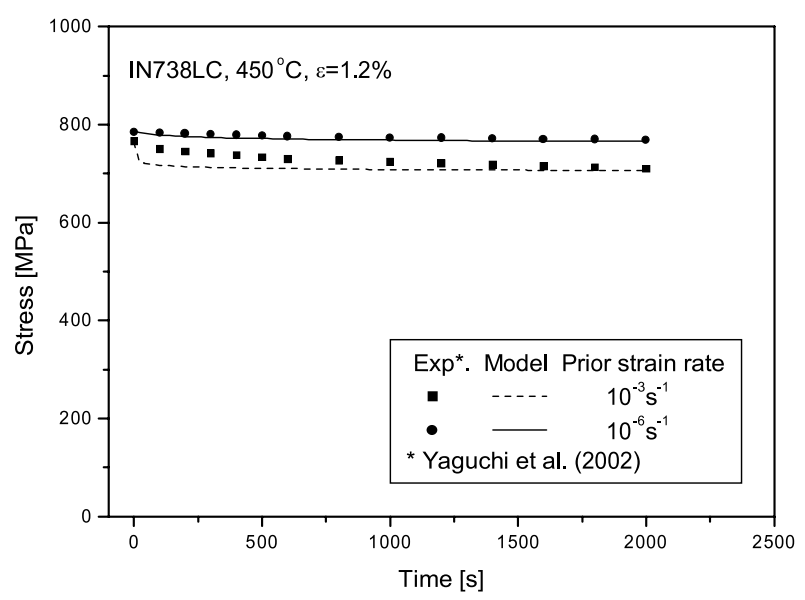

(b)

Fig. 10 Simulations of superalloy IN738LC at $450^{\circ} \mathrm{C}$ : (a) stress-strain curve, (b) relaxation behavior

ries, nonlinear kinematic hardening rules of the Frederick and Armstrong type have been used and modified as the evolution laws of state variables, which take the rateindependent format, in most of viscoplastic constitutive models. In contrast, the proposed model introduces the rate dependency in the evolution law of the back stress that is a state variable in the constitutive equations. This property admits to model negative rate sensitivity related to dynamic strain aging and the nonlinear dependence of relaxation behavior on prior strain rate.

The basic features of the model are well explained with the help of the long-term asymptotic solutions corresponding to the fully established inelastic flow. For the asymptotic state, the flow stress is expressed as the sum of the rate-independent contribution and the rate-dependent one. Consequently, the three types of rate sensitivity, namely positive, zero and negative rate sensitivities, can be mathematically classified with respect to the model parameter $\beta$ by controlling the contribution of the ratedependent term to the flow stress.

The numerical evaluations using the arbitrarily cho- sen material constants demonstrated the various modeling capabilities of the proposed model. And the applications to the inelastic deformation of modified $9 \mathrm{Cr}-1 \mathrm{Mo}$ steel and superalloy IN738LC showed good correlation with the experimental data reported in the literature.

\section{References}

(1) Bodner, S.R. and Partom, Y., Constitutive Equations for Elastic-Viscoplastic Strain Hardening Materials, J. Appl. Mech., Vol.42 (1975), pp.385-389.

( 2 ) Miller, A., An Inelastic Constitutive Model for Monotonic, Cyclic and Creep Deformations, J. Eng. Mat. Tech., Vol.98 (1976), pp.97-105.

( 3 ) Chaboche, J.L. and Rousselier, G., On the Plastic and Viscoplastic Constitutive Equations. Part I: Rules Developed with Internal Variable Concept, J. Pres. Vessel Tech., Vol.105 (1983), pp.153-158.

( 4 ) Krempl, E., Models of Viscoplasticity: Some Comments on Equilibrium (Back) Stress and Drag Stress, Acta Mech., Vol.69 (1987), pp.25-42.

( 5 ) Ohno, N. and Wang, J., Kinematic Hardening Rules with Critical State of Dynamic Recovery, Part I: Formulation and Basic Features for Ratcheting Behavior, Int. J. Plasticity, Vol.9 (1993), pp.375-390.

( 6 ) Cottrell, A.H., A Note on the Portevin-Le Chatelier Effect, Phil. Mag., Vol.44 (1953), pp.829-832.

( 7 ) Mulford, R.A. and Kocks, U.F., New Observation on the Mechanisms of Dynamic Strain Aging and of Jerky Flow, Acta Metal., Vol.27 (1979), pp.1125-1134.

( 8 ) Kubin, L.P. and Estrin, Y., The Portevin-Le Chatelier Effect in Deformation with Constant Stress Rate, Acta Metal., Vol.33 (1985), pp.397-407.

(9) Nortmann, A. and Schwink, CH., Characteristics of Dynamic Strain Ageing in Binary F.C.C. Copper Alloys: I. Results on Solid Solutions of CuAl, Acta Mat., Vol.45 (1997), pp.2043-2050.

(10) Miller, A.K. and Sherby, O.D., A Simplified Phenomenological Model for Non-Elastic Deformation: Prediction of Pure Aluminum Behavior and Incorporation of Solute Strengthening Effects, Acta Metal., Vol.26 (1978), pp.289-304.

(11) Yaguchi, M. and Takahashi, Y., Unified Inelastic Constitutive Model for Modified 9Cr-1Mo Steel Incorporating Dynamic Strain Aging Effect, JSME Int. J., Ser. A, Vol.42, No.1 (1999), pp.1-10.

(12) Perzyna, P., The Constitutive Equations for Rate Sensitive Plastic Materials, Quart. Appl. Math., Vol.20 (1963), pp.321-332.

(13) Chaboche, J.L., Constitutive Equations for Cyclic Plasticity and Cyclic Viscoplasticity, Int. J. Plasticity, Vol.5 (1989), pp.247-302.

(14) Ho, K. and Krempl, E., The Modeling of Unusual Rate Sensitivity Inside and Outside the Dynamic Strain Aging Regime, J. Eng. Mat. Tech., Vol.123 (2001), pp.2835.

(15) Ho, K., Modeling of Nonlinear Rate Sensitivity by Using an Overstress Model, Comp. Model. Eng. Sci., Vol.2 (2001), pp.351-364.

(16) Armstrong, P.J. and Frederick, C.O., A Mathematical Representation of the Multiaxial Bauschinger Effect, 
CEGB Report RD/B/N731, Berkeley Nuclear Lab., UK., (1966).

(17) Cernocky, E.P. and Krempl, E., A Nonlinear Uniaxial Integral Constitutive Equation Incorporating Rate Effects, Creep and Relaxation, Int. J. Nonlinear Mech., Vol.14 (1979), pp.183-203.

(18) Majors, P.S. and Krempl, E., The Isotropic Viscoplasticity Theory Based on Overstress Applied to the Modeling of Modified $9 \mathrm{wt} . \% \mathrm{Cr}-1 \mathrm{wt} . \%$ Mo Steel at $538^{\circ} \mathrm{C}$, Mat. Sci. Eng., Vol.186 (1994), pp.23-34.

(19) Krempl, E. and Nakamura, T., The Influence of the Equilibrium Stress Growth Law Formulation on the Modeling of Recently Observed Relaxation Behaviors, JSME Int. J., Ser. A, Vol.41, No.1 (1998), pp.103-111.

(20) McCormick, P.G., Theory of Flow Localisation Due to Dynamic Strain Aging, Acta Metal., Vol.36 (1988), pp.3061-3067.

(21) Estrin, Y. and Kubin, L.P., Plastic Instabilities: Phenomenology and Theory, Mat. Sci. Eng. A, Vol.137 (1991), pp.125-134.

(22) Mesarovic, S., Dynamic Strain Aging and Plastic Instabilities, J. Mech. Phys. Solids, Vol.43 (1995), pp.671700 .

(23) Kaneko, K. and Oyamada, T., A Viscoplastic Constitutive Model with Effect of Aging, Int. J. Plasticity, Vol.16 (2000), pp.337-357.

(24) Yaguchi, M., Yamamoto, M. and Ogata, T., A Viscoplastic Constitutive Model for Nickel-Base Superalloy, Part2: Modeling under Anisothermal Conditions, Int. J. Plasticity, Vol.18 (2002), pp.1111-1131. 